\title{
ITERATIVE DEFECT CORRECTION AND MULTIGRID ACCELERATED EXPLICIT TIME STEPPING SCHEMES FOR THE STEADY EULER EQUATIONS*
}

\author{
MARIE-HÉLÈNE LALLEMAND $\dagger$ AND BARRY KOREN $\ddagger$
}

\begin{abstract}
Analytical and experimental convergence results are presented for a novel pseudo-unsteady solution method for higher-order accurate upwind discretizations of the steady Euler equations. Comparisons are made with an existing pseudo-unsteady solution method. Both methods make use of nonlinear multigrid for acceleration and nested iteration for the fine-grid initialization. The new method uses iterative defect correction. Analysis shows that it not only has better stability but it also has better smoothing properties. The analytical results are confirmed by numerical experiments, which show better convergence and efficiency.
\end{abstract}

Key words. defect correction, multigrid, explicit time stepping, Euler equations

AMS subject classifications. 65B05, 65N10, 65N20, 65N30, 65N40, 76G15

1. Introduction.

1.1. Equations. The equations considered are the steady, two-dimensional, compressible Euler equations

$$
\frac{\partial F(W)}{\partial x}+\frac{\partial G(W)}{\partial y}=0
$$

where

$$
W=\left(\begin{array}{c}
\rho \\
\rho u \\
\rho v \\
\rho e
\end{array}\right), \quad F(W)=\left(\begin{array}{c}
\rho u \\
\rho u^{2}+p \\
\rho u v \\
\rho u(e+p / \rho)
\end{array}\right), \quad G(W)=\left(\begin{array}{c}
\rho v \\
\rho v u \\
\rho v^{2}+p \\
\rho v(e+p / \rho)
\end{array}\right) \text {. }
$$

Assuming a perfect gas, the total energy $e$ satisfies $e=p /(\rho(\gamma-1))+\frac{1}{2}\left(u^{2}+v^{2}\right)$. The ratio of specific heats $\gamma$ is assumed to be constant.

1.2. Spatial discretization. The computational grid is obtained by a hybrid finite element-finite volume partition. A (possibly unstructured) finite element triangularization is used as the basic partition. A cell-centered finite volume partition is derived from the finite element partition by connecting the centers of the triangle sides in the manner illustrated in Fig. 1. The finite volume grid gives us the easy possibility of grouping together the nodes associated with contiguous finite volumes. If we take unions of control volumes this results in a new coarser mesh. Repetition of this operation gives coarser and coarser meshes. For details about this hybrid way of constructing finite volume grids, see [1]. For applications in single-grid Euler and Navier-Stokes flow computations, we refer to [5] and [23], respectively. For details about the coarsening process (multilevel gridding), we refer to [16].

* Received by the editors April 17, 1989; accepted for publication (in revised form) August 4, 1992. This work was performed as a cooperation between l'Institut National de Recherche en Informatique et en Automatique (INRIA) and the Centrum voor Wiskunde en Informatica (CWI). The investigations were supported by the Centre National d'Etudes Spatiales (CNES), and by the European Space Agency (ESA) through Avions Marcel Dassault-Bréguet Aviation (AMD-BA).

$\dagger$ L'Institut National de Recherche en Informatique et en Automatique, Domaine de Voluceau, Rocquencourt, B.P. 105, 78153 Le Chesnay Cedex, France.

$\ddagger$ Centrum voor Wiskunde en Informatica, P.O. Box 4079, 1009 AB Amsterdam, the Netherlands. 


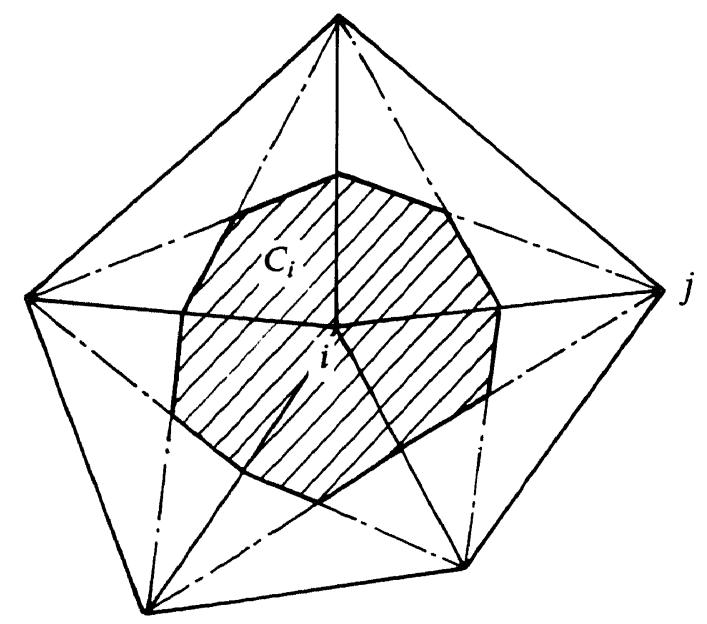

FIG. 1. Finite volume $C_{i}$.

On the finest grid, for all finite volumes $C_{i}, i=1,2, \ldots, N$, we consider the integral form

$$
\oint_{\partial C_{i}}\left(F(W) n_{x}+G(W) n_{y}\right) d s=0, \quad i=1,2, \ldots, N
$$

with $n_{x}$ and $n_{y}$ the $x$ - and $y$-component of the outward unit normal on the volume boundary $\partial C_{i}$. For the Euler equations, because of their rotational invariance, (1.3) may be rewritten as

$$
\oint_{\partial c_{i}} T^{-1}\left(n_{x}, n_{y}\right) F\left(T\left(n_{x}, n_{y}\right) W\right) d s=0, \quad i=1,2, \ldots, N,
$$

where $T\left(n_{x}, n_{y}\right)$ is the rotation matrix

$$
T\left(n_{x}, n_{y}\right)=\left(\begin{array}{cccc}
1 & 0 & 0 & 0 \\
0 & n_{x} & n_{y} & 0 \\
0 & -n_{y} & n_{x} & 0 \\
0 & 0 & 0 & 1
\end{array}\right) .
$$

For simplicity, we assume the flux to be constant across each bisegment $\partial C_{i j}$ of the boundary $\partial C_{i}$, where $\partial C_{i j}=\partial C_{i} \cap \partial C_{j}$ is the common boundary between the neighboring volumes $C_{i}$ and $C_{j}$ (Fig. $2(\mathrm{a})$ ). Hence, $\partial C_{i}=\bigcup \partial C_{i j}, j=1,2, \ldots, n_{i}$, with $n_{i}$ the number of neighboring volumes $C_{j}$. (In the example of Fig. $1 ; n_{i}=5$.) Since we have assumed that the flux is constant along $\partial C_{i j}$, it is equal to the flux across the straight segment

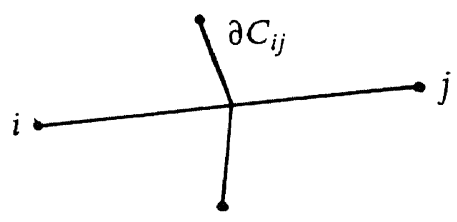

(a)

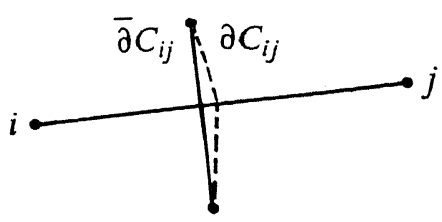

(b)

FIG. 2. Segments in between finite volumes $C_{i}$ and $C_{j}$. (a) Bisegment $\partial C_{i j}$. (b) Straight segment $\bar{\partial} C_{i j}$. 
$\bar{\partial} C_{i j}$ connecting the two extreme points of $\partial C_{i j}$ (Fig. 2(b)). If we introduce the outward unit normal $\bar{n}_{i j}=\left(\left(\bar{n}_{x}\right)_{i j},\left(\bar{n}_{y}\right)_{i j}\right)^{T}$ along each $\bar{\partial} C_{i j}, j=1,2, \ldots, n_{i}$, with the assumption of a constant flux, the contour integral (1.4) can be rewritten as the sum

$$
\sum_{j=1}^{n_{i}} \bar{T}_{i j}^{-1} F\left(\bar{T}_{i j} W_{i j}\right) l_{i j}=0, \quad i=1,2, \ldots, N,
$$

where $\bar{T}_{i j}=T\left(\left(\bar{n}_{x}\right)_{i j},\left(\bar{n}_{y}\right)_{i j}\right)$, where $W_{i j}$ is some value of $W$ depending on for instance $W_{i}$ and $W_{j}$, and where $l_{i j}$ is the length of the segment $\bar{\partial} C_{i j}$.

Crucial in (1.6) is the way in which the cell face flux $F\left(\bar{T}_{i j} W_{i j}\right)$ is evaluated. For this we use an upwind scheme that follows the Godunov principle [8], which assumes that the constant flux vector along each segment $\bar{\partial} C_{i j}$ is determined only by a uniformly constant left and right cell face state $\left(W_{i j}^{l}\right.$ and $\left.W_{i j}^{r}\right)$. The one-dimensional Riemann problem, which then arises at each cell face, is solved in an approximate way. With this, (1.6) can be further written as

$$
\sum_{j=1}^{n_{i}} \bar{T}_{i j}^{-1} \Phi\left(\bar{T}_{i j} W_{i j}^{l}, \bar{T}_{i j} W_{i j}^{r}\right) l_{i j}=0, \quad i=1,2, \ldots, N,
$$

where $\Phi$ denotes the approximate Riemann solver. Several approximate Riemann solvers exist (see, for example, [20] and [22]). In this paper, without any particular motivation, we restrict ourselves to the application of Osher's approximate Riemann solver [20].

The flux evaluation, and so the space discretization, may be either first- or higher-order accurate. First-order accuracy is obtained in the standard way; at each finite volume wall, the left and right cell face state, which must be inserted in the numerical flux function, are taken equal to those in the corresponding adjacent volumes

$$
\begin{aligned}
& W_{i j}^{l}=W_{i}, \\
& W_{i j}^{r}=W_{j} .
\end{aligned}
$$

Whereas the first-order accurate discretization is applied at all levels, the higher-order discretization is applied at the finest grid only, using the finite element partition existing there. Higher-order accuracy is obtained with a MUSCL approach [18]. Here, $W_{i j}^{l}$ and $W_{i j}^{r}$ are derived from linear interpolations. On each volume $C_{i}$ around the triangle-vertex $i$ an approximate gradient, denoted by $(\bar{\nabla} W)_{i}$, is derived by integrating the gradient of the linear interpolant of $W$ over all the triangles that have $i$ as a vertex:

$$
(\bar{\nabla} W)_{i}=\left[\left(\frac{\bar{\partial} W}{\partial x}\right)_{i},\left(\frac{\bar{\partial} W}{\partial y}\right)_{i}\right]^{T}
$$

with

$$
\begin{aligned}
\left(\frac{\partial}{\partial} W\right. & )_{i}=\frac{\int_{\text {supp }(i)}(\partial W / \partial x) d x d y}{\int_{\operatorname{supp}(i)} d x d y}, \\
\left(\frac{\partial}{\partial y}\right)_{i} & =\frac{\int_{\text {supp }(i)}(\partial W / \partial y) d x d y}{\int_{\text {supp }(i)} d x d y} .
\end{aligned}
$$

Here, supp ( $i$ ) denotes the union of triangles which have $i$ as a vertex. Then for each pair of neighboring vertices $(i, j)$ we compute the extrapolated values

$$
\begin{aligned}
& W_{i j}^{l}=W_{i}+\frac{1}{2}(\bar{\nabla} W)_{i} \cdot \overline{i j}, \\
& W_{i j}^{r}=W_{j}-\frac{1}{2}(\bar{\nabla} W)_{j} \cdot \overline{i j} .
\end{aligned}
$$


On equidistant grids, this higher-order accurate discretization can be formally proved to be second-order accurate. The proof is still valid for nearly equidistant grids. In this paper we do not analyze orders of accuracy; the discretization is already known. It has been described in more detail in other papers; see, for example, [7].

In order to ensure monotonicity, while preserving the higher-order accuracy in smooth flow regions, the higher-order values $W_{i j}^{l}$ and $W_{i j}^{r}$ according to (1.10) can be replaced by limited values that do not affect the order of accuracy.

1.3. Existing solution method. To solve the steady discretized system (1.7), we consider the unsteady, semidiscrete system of ordinary differential equations

$$
\frac{d W_{i}}{d t}=R_{i}, \quad i=1,2, \ldots, N .
$$

The natural choice for $R_{i}$ is

$$
R_{i}=\frac{-1}{A_{i}} \sum_{j=1}^{n_{i}} \bar{T}_{i j}^{-1} \Phi\left(\bar{T}_{i j} W_{i j}^{l}, \bar{T}_{i j} W_{i j}^{r}\right) l_{i j},
$$

where $A_{i}$ is the area of finite volume $C_{i}$.

As an upwind analogue to Jameson's central method [13], in [16] and [17] an explicit four-stage Runge-Kutta (RK4) scheme is applied for the temporal integration of (1.11)-(1.12). The benefits of the upwind analogue are evident: better shock capturing, greater robustness, and no tuning of explicitly added artificial viscosity. Similarly, just as in [13], in [16], multigrid is applied for accelerating the solution process. Furthermore, just as in [13], time accuracy is not pursued, and optimal Runge-Kutta coefficients are applied to get good stability as well as good smoothing properties. It seems that the solution method presented in [16] is already competitive with Jameson's method, without the introduction of a further acceleration technique such as, for example, residual averaging.

It is interesting that the upwind analogue allows a further efficiency improvement by exploitation of the direct availability of the corresponding first-order upwind discretization, with its better stability and smoothing properties. Since a first-order central discretization is not readily available, a standard central method does not easily allow this improvement.

\section{Novel solution method.}

2.1. Explicit time stepping. Compared with the existing solution method, the new solution method only uses a more extensive right-hand side in the explicit time stepping scheme. The extension consists of two first-order upwind defects; one which is evaluated at each stage of the multistage scheme, and another which is kept frozen during a fixed number of $\nu_{t}$ RK4 time steps $\left(\nu_{t} \geqq 1\right)$, and which compensates for the other first-order defect by its opposite sign. Furthermore, significantly, the higher-order defect is kept frozen as well during $\nu_{t}$ RK4 steps. The four-stage time stepping scheme is written as

$$
\begin{aligned}
& W_{i}^{0,4}:=W_{i}^{0,0}, \quad i=1,2, \ldots, N \\
& \text { for } \nu \text { from } 1 \text { to } \nu_{t} \text { do } \\
& \quad W_{i}^{\nu, 0}:=W_{i}^{\nu-1,4}, \quad i=1,2, \ldots, N \\
& \quad \text { for } k \text { from } 1 \text { to } 4 \text { do } \\
& \quad W_{i}^{\nu, k}:=W_{i}^{\nu, 0}+\Delta t_{i} \alpha_{k} R_{i}^{\nu, k-1}, \quad i=1,2, \ldots, N \\
& \text { enddo } \\
& \text { enddo }
\end{aligned}
$$


Here, $\nu$ is the time step number, $k$ the stage number, $\Delta t_{i}$ the local time step, and $\alpha_{k}$ the $k$ th Runge-Kutta coefficient. In the existing higher-order method the right-hand side $R_{i}^{\nu, k-1}$ is

$$
R_{i}^{\nu, k-1}=\frac{-1}{A_{i}} \sum_{j=1}^{n_{i}} \bar{T}_{i j}^{-1} \Phi\left(\bar{T}_{i j}\left(W_{i j}^{l}\right)^{\nu, k-1}, \bar{T}_{i j}\left(W_{i j}^{r}\right)^{\nu, k-1}\right) l_{i j},
$$

with $\left(W_{i j}^{l}\right)^{\nu, k-1}$ and $\left(W_{i j}^{r}\right)^{\nu, k-1}$ higher-order accurate. So, nothing is kept frozen in the existing method's right-hand side. For the novel method, we take

$$
\begin{aligned}
R_{i}^{\nu, k-1}=\frac{-1}{A_{i}} \sum_{j=1}^{n_{i}} \bar{T}_{i j}^{-1}( & \Phi\left(\bar{T}_{i j} W_{i}^{\nu, k-1}, \bar{T}_{i j} W_{j}^{\nu, k-1}\right)-\Phi\left(\bar{T}_{i j} W_{i}^{0,0}, \bar{T}_{i j} W_{j}^{0,0}\right) \\
+ & \left.\Phi\left(\bar{T}_{i j}\left(W_{i j}^{l}\right)^{0,0}, \bar{T}_{i j}\left(W_{i j}^{r}\right)^{0,0}\right)\right) l_{i j},
\end{aligned}
$$

where only $\left(W_{i j}^{l}\right)^{0,0}$ and $\left(W_{i j}^{r}\right)^{0,0}$ are higher-order accurate. The frozen first-order cell face states $\left(W_{i}^{0,0}\right.$ and $\left.W_{j}^{0,0}\right)$ and the frozen higher-order cell face states $\left(\left(W_{i j}^{l}\right)^{0,0}\right.$ and $\left.\left(\boldsymbol{W}_{i j}^{r}\right)^{0,0}\right)$ are updated in an additional outer iteration, which will be explained in the next section. In the following, for convenience, $W^{\nu, 4}$ will also be denoted as $W_{\mathrm{RK} 4}\left(\nu, R^{0,0}, W^{0,0}\right)$.

2.2. Complete solution method. The novel solution method is of defect correction type [2]. Though defect correction iteration is not as necessary for a pseudo-unsteady solution method as it is for a solution method that directly tackles steady discretized equations [12], [14], [15], it may lead to an improved efficiency. In $\S 3$, we will show that the defect correction method proposed here can take advantage of a greater stability domain (larger local time steps) guaranteed by the first-order defects in the right-hand side. Furthermore, we will show that with multigrid as an acceleration technique, advantage can also be taken of better smoothing properties.

The new solution method can be divided into two successive stages. The first stage is nested iteration [10, p.98], also called full multigrid (FMG) method [4], which is applied to obtain a good initial solution on the finest grid. The second stage is an iterative defect correction (IDeC) method [2], [10, p. 282], which is used to iterate until the higher-order accurate solution is obtained. The initial solution for the defect correction process is the solution obtained by the nested iteration. The inner iteration of both stages is a nonlinear multigrid method [10, p. 181], viz. the full approximation storage (FAS) algorithm [3], [4]. In the following sections we discuss successively: the nested iteration $(\S 2.2 .1)$, the iterative defect correction method $(\S 2.2 .2)$, and the building block of these two iterations: the nonlinear multigrid iteration $(\$ 2.2 .3)$.

2.2.1. Nested iteration. To apply multigrid we construct a nested set of grids. Let $\Omega_{1}, \Omega_{2}, \ldots, \Omega_{L}$ be a sequence of nested grids with $\Omega_{1}$ the coarsest and $\Omega_{L}$ the finest grid. (For a description of the coarsening rule applied here, we refer to [16].) The nested iteration (FMG) starts with a user-defined initial estimate of $W_{1}$ : the solution on the coarsest grid $\Omega_{1}$. To obtain an initial solution on $\Omega_{2}$, the solution on $\Omega_{1}$ is first improved by a few FAS cycles. (The number of FAS cycles that is applied in each FMG step can be either fixed, $\nu_{\text {FAS }}=$ constant, or dependent on the residual.) After this, the improved solution $W_{1}$ is prolongated to $\Omega_{2}$. The process is repeated until $\Omega_{L}$ has been reached.

The prolongation of the solution can be the simple piecewise constant prolongation $I_{l-1}^{l}, 2 \leqq l \leqq L$, or it can be a smoother one. If we denote the area of finite volume $C_{i}^{l}$ at level $l$ by $A_{i}^{l}$, and the number of neighboring volumes $C_{j}^{l}$ of $C_{i}^{l}$ by $n_{i}^{l}$, a smooth 
prolongation operator $\mathscr{I}_{l-1}^{l}$ is defined by

$$
\left(\mathscr{f}_{l-1}^{l}\left(W_{l-1}\right)\right)_{i} \equiv \frac{A_{i}^{l}\left(I_{l-1}^{l}\left(W_{l-1}\right)\right)_{i}+\sum_{j=1}^{n l} A_{j}^{l}\left(I_{l-1}^{l}\left(W_{l-1}\right)\right)_{j}}{A_{i}^{l}+\sum_{j=1}^{n_{i}^{\prime}} A_{j}^{l}}, \quad 2 \leqq l \leqq L .
$$

We note that since $I_{l-1}^{l}$ strictly obeys the physical conservation laws by the prolongation of cell-integrated amounts of mass, momentum, and energy, $\mathscr{I}_{l-1}^{l}$ is strictly conservative as well.

2.2.2. Iterative defect correction. Let $\mathscr{F}_{L}^{1}\left(W_{L}\right)=0$ and $\mathscr{F}_{L}^{+}\left(W_{L}\right)=0$ denote the first-order and higher-order discretized Euler equations, respectively, on the finest grid. Then, IDeC can be written as

$$
\begin{aligned}
& \mathscr{F}_{L}^{1}\left(W_{L}^{0}\right)=0, \\
& \mathscr{F}_{L}^{1}\left(W_{L}^{n}\right)=\mathscr{F}_{L}^{1}\left(W_{L}^{n-1}\right)-\mathscr{F}_{L}^{+}\left(W_{L}^{n-1}\right), \quad n=1,2, \ldots, n_{\mathrm{IDeC}},
\end{aligned}
$$

where $W_{L}^{0}$ is the solution yielded by FMG. From (2.5b), it is immediately clear that at convergence $\left(W_{L}^{n}=W_{L}^{n-1}=W_{L}\right)$, we have solved the higher-order discretized Euler equations $\mathscr{F}_{L}^{+}\left(W_{L}\right)=0$. Therefore, we emphasize that the present defect correction method is not mixed defect correction iteration [11]. (A mixed defect correction method would yield a solution whose accuracy is not well defined; its solution would be a vague mixture of the first-order and higher-order accurate solutions.) Though both theory [6] and practice [6] show that IDeC gives poor convergence of the residual, theory [9], [10, p. 282] and practice [12], [15] also show that for smooth problems, a single IDeC cycle $\left(n_{\mathrm{IDeC}}=1\right)$ is sufficient to obtain second-order solution accuracy. Furthermore, for solutions with discontinuities, a few IDeC cycles $\left(n_{\mathrm{IDec}} \approx 5\right)$ may improve the accuracy to a sufficient extent [12], [14]. In summary, for both smooth and nonsmooth flow problems, numerical experiments with IDeC show this phenomenon of slow convergence but of fast solution improvement [6], [12], [14], [15]; a phenomenon that is understood by theory [6], [9], [10, p. 282].

In each IDeC cycle we must solve a first-order system with an appropriate right-hand side. From [14] it is known that it is inefficient to solve this system very accurately. With a steady approach, application of only a single FAS cycle per IDeC cycle appears to be the most efficient strategy in [14]. In this paper, with our unsteady approach, we will re-investigate what is the most efficient number of FAS cycles per IDeC cycle (see $\S 3$ ).

2.2.3. Nonlinear multigrid iteration. Let us denote by $\left(W_{l}\right)_{V\left(\nu_{\text {pre }}, \nu_{\text {post }}\right)}\left(\nu_{\mathrm{FAS}}, R_{l}, W_{l}^{0}\right)$ the solution obtained on level $l$, after $\nu_{\mathrm{FAS}}$ FAS $V\left(\nu_{\text {pre }}, \nu_{\text {post }}\right)$-cycles have been applied to $\mathscr{F}_{l}^{1}\left(W_{l}\right)=R_{l}$, with initial solution $W_{l}^{0}$. A single FAS $V\left(\nu_{\text {pre }}, \nu_{\text {post }}\right)$-cycle on level $l, 1 \leqq l \leqq L$, is then recursively defined by the following successive steps:

(i) Improve on the grid $\Omega_{l}$ the initial solution $W_{l}^{0}$ by applying $\nu_{\text {pre }}$ RK4 steps to

$$
\mathscr{F}_{l}^{1}\left(W_{l}\right)=R_{l} \text {. }
$$

Let us denote the resulting solution $\left(W_{l}\right)_{\mathrm{RK} 4}\left(\nu_{\mathrm{pre}}, R_{l}, W_{l}^{0}\right)$ by $\bar{W}_{l}$.

(ii) Coarse-grid correction step: Approximate on the underlying coarser grid $\Omega_{l-1}$ the solution of

$$
\begin{gathered}
\mathscr{F}_{l-1}^{1}\left(W_{l-1}\right)=R_{l-1}, \\
R_{l-1}=\mathscr{F}_{l-1}^{1}\left(I_{l}^{l-1}\left(\bar{W}_{l}\right)\right)-\tilde{I}_{l}^{l-1}\left(\mathscr{F}_{l}^{1}\left(\bar{W}_{l}\right)-R_{l}\right),
\end{gathered}
$$

by applying a single FAS $V\left(\nu_{\text {pre }}, \nu_{\text {post }}\right)$-cycle on level $l-1$. Let us denote the resulting approximate solution $\left(W_{l-1}\right)_{V\left(\nu_{\text {pre }}, \nu_{\text {pos } 1}\right)}\left(1, R_{l-1}, I_{l}^{l-1}\left(\bar{W}_{l}\right)\right)$ by $\overline{\mathrm{W}}_{l-1}$. 
(iii) Improve the solution on $\Omega_{l}$ by first correcting the approximate solution $\bar{W}_{l}$ obtained in step (i):

$$
\bar{W}_{l}:=\bar{W}_{l}+I_{l-1}^{l}\left(\Delta W_{l-1}\right) \text {, }
$$

where $\Delta W_{l-1}=\bar{W}_{l-1}-I_{l}^{l-1} \bar{W}_{l}$ is the result of the coarse-grid correction step (ii). Furthermore, improve $\bar{W}_{l}$ by applying $\nu_{\text {post }}$ RK4 steps to $(2.6): W_{l}:=\left(W_{l}\right)_{\mathrm{RK} 4}\left(\nu_{\text {post }}, R_{l}, \bar{W}_{l}\right)$.

In the FMG-stage in step (i), we have on each starting (locally finest) grid $\Omega_{l}, 1 \leqq l \leqq L: R_{l}=0$. Hence, the initial solution for IDeC as obtained by FMG is at most first-order accurate. In the IDeC stage the starting grid is always the globally finest grid $\Omega_{L}$, and there we have: $R_{L}=\mathscr{F}_{L}^{1}\left(W_{L}\right)-\mathscr{F}_{L}^{+}\left(W_{L}\right)$. This higher-order right-hand side is kept frozen during $\nu_{\mathrm{FAS}}$ FAS $V\left(\nu_{\text {pre }}, \nu_{\text {post }}\right)$-cycles per IDeC cycle. Note that with the novel method, we evaluate the higher-order operator at most once per FAS $V\left(\nu_{\text {pre }}, \nu_{\text {post }}\right)$-cycle, instead of $4 \times\left(\nu_{\text {pre }}+\nu_{\text {post }}\right)+1$ times per FAS $V\left(\nu_{\text {pre }}, \nu_{\text {post }}\right)$-cycle with the existing method.

In step (ii), note that in the RK4 scheme, the complete right-hand side $R_{l-1}$ is kept frozen. Just as the prolongation operator $I_{l-1}^{l}$, the restriction operator $I_{l}^{I-1}$ is such that it also exactly obeys the conservation of cell-integrated mass, momentum, and energy. The restriction operator $\tilde{I}_{l}^{l-1}$ restricts the defect in the standard way; by summation of mass, momentum, and energy defects over fine-grid cells whose union is a coarse cell. On the coarsest grid $\left(\Omega_{1}\right)$, step (ii) (the coarse-grid correction step) is skipped of course.

To illustrate the structure of the complete novel solution method, we give two examples of a complete higher-order solution schedule in Fig. 3. The schedule in Fig. 3(a) is fixed by $L=2, \nu_{\text {pre }}=1, \nu_{\text {post }}=2, \nu_{\mathrm{FAS}}=2, n_{\mathrm{IDeC}}=2$. The schedule in Fig. 3(b) is fixed by $L=3, \nu_{\text {pre }}=\nu_{\text {post }}=1, \nu_{\mathrm{FAS}}=1, n_{\mathrm{IDeC}}=3$. In both figures, the marker $\triangleright$ denotes a single RK4 step (over $\Omega_{l}$ ) preceding a coarse-grid correction, whereas the marker $\triangleleft$ denotes a single RK4 step after the coarse-grid correction. The marker $\bigcirc$ denotes the computation of $R_{L}=\mathscr{F}_{L}^{l}\left(W_{L}\right)-\mathscr{F}_{L}^{+}\left(W_{L}\right)$. Note that the corresponding first-order variants of both schedules (i.e., the variants without any marker $O$ ) are simply obtained by taking $n_{1 \mathrm{DeC}}=0$.

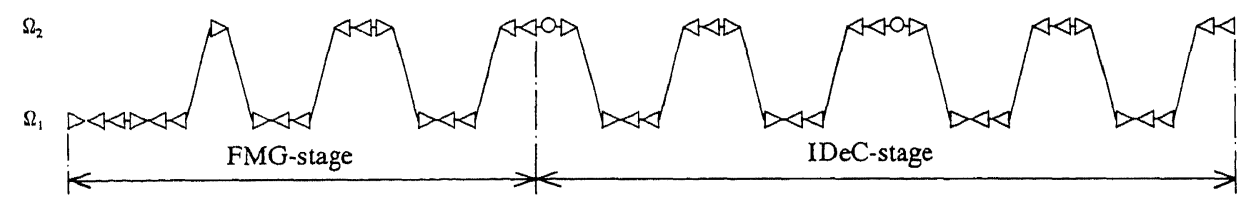

(a)

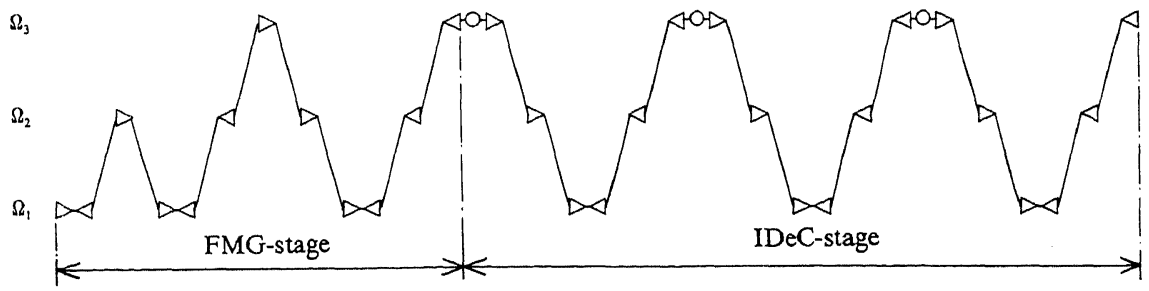

(b)

FIG. 3. Examples of complete solution schedule. (a) $L=2, \nu_{\text {pre }}=1, \nu_{\text {post }}=2, \nu_{\mathrm{FAS}}=2, n_{\mathrm{IDeC}}=2$. (b) $L=3$, $\nu_{\text {pre }}=\nu_{\text {post }}=1, \nu_{\text {FAS }}=1, n_{\mathrm{IDeC}}=3$. 
2.3. Analysis. To analyze the convergence properties of the $I D e C$ method proposed, we consider the unsteady, linear, scalar, one-dimensional model equation

$$
\frac{\partial w}{\partial t}+c \frac{\partial w}{\partial x}=0, \quad c>0 .
$$

For the spatial discretization, we consider a grid with a uniformly constant mesh size $h$. Then we have, for fixed time $t$, for the first-order upwind discretization,

$$
\frac{\partial w}{\partial x}=\frac{-w_{i-1}+w_{i}}{h}+O(h) \text {. }
$$

As a higher-order upwind discretization, we take the Fromm scheme (i.e., van Leer's $\kappa$-scheme [19] with $\kappa=0$ ), which leads to

$$
\frac{\partial w}{\partial x}=\frac{w_{i-2}-5 w_{i-1}+3 w_{i}+w_{i+1}}{4 h}+O\left(h^{2}\right) .
$$

For these two spatial discretizations and the existing explicit solution method, the following Runge-Kutta coefficients can be derived by maximizing the maximally allowable CFL number: $\alpha_{1}=0.11, \alpha_{2}=0.2767, \alpha_{3}=0.5$ (see [17]). Consistency requires $\alpha_{4}=1$. The optimization can be redone for our new solution method. However, in the next section we will show that if we simply omit the optimization, the new method already yields both better stability and better smoothing with the $\alpha_{k}$ 's given above (i.e., the $\alpha_{k}$ 's found for the existing higher-order method).

2.3.1. Stability analysis. First we will perform a stability analysis for IDeC with an explicit RK4 scheme as the inner solution method. Let us denote the steady-state analogue of (2.9) by

$$
A w=c \frac{d w}{d x}
$$

Assuming that we have periodic boundary conditions, we denote by $\boldsymbol{A}_{1}$ and $\boldsymbol{A}_{+}$the first-order and higher-order accurate, linear operators, approximating $A$. Then, the IDeC process to solve the discrete linear system

$$
A_{+} W=0
$$

can be written as

$$
\begin{gathered}
A_{1} W^{0}=0, \\
A_{1} W^{n}=\left(A_{1}-A_{+}\right) W^{n-1}, \quad n=1,2, \ldots, n_{\mathrm{IDeC}},
\end{gathered}
$$

where $A_{1}$ denotes the matrix resulting from the first-order discretization. Assuming $A_{1}$ to be invertible, the corresponding amplification matrix $M$ reads

$$
M=I-A_{1}^{-1} A_{+} .
$$

From (2.15) it is clear that to have convergence of IDeC, the spectral radius of $M$ should be smaller than one. It is also clear that the better the resemblance between $A_{1}$ and $A_{+}$, the faster the convergence of IDeC. (Also important for good efficiency of IDeC is, of course, that $A_{1}$ can be inverted in an efficient way.)

Instead of solving each $W^{n}$ from $(2.14 \mathrm{~b})$ exactly, we approximate it by means of the explicit RK4 scheme (2.1) with

$$
\begin{gathered}
W^{0,0}=W^{n-1}, \\
R^{\nu, k-1}=-\left(A_{1} W^{\nu, k-1}-\left(A_{1}-A_{+}\right) W^{0,0}\right) .
\end{gathered}
$$


With $\nu$ the number of RK4 steps which allows us to define an (approximate) solution $W^{n}$, we now investigate how the corresponding amplification matrix $M_{v}$ is related to the amplification matrix $M$, which corresponds with the exact solution of $W^{n}$ from (2.14b). If we consider the approximate solution $W^{\nu, 4}$, for $\nu=1$ we have

$$
W^{1,4}=\left(P+Q\left(A_{1}-A_{+}\right)\right) W^{n-1},
$$

with

$$
\begin{gathered}
P=P\left(-\Delta t A_{1}\right), \\
Q=I-A_{1}^{-1} P, \\
P(Z)=I+Z+\alpha_{3} Z^{2}+\alpha_{3} \alpha_{2} Z^{3}+\alpha_{3} \alpha_{2} \alpha_{1} Z^{4} .
\end{gathered}
$$

We can easily verify that $A_{1} Q=Q A_{1}$, and that $A_{1}^{-1} P=P A_{1}^{-1}$. Hence, for $M_{1}$ we can write

$$
M_{1}=P+Q\left(A_{1}-A_{+}\right) .
$$

For $\nu>1$, we can easily find the recurrence relation

$$
M_{\nu}=P M_{\nu-1}+Q\left(A_{1}-A_{+}\right),
$$

which leads to

$$
M_{\nu}=\left(I+P+P^{2}+\cdots+P^{\nu-1}\right) Q\left(A_{1}-A_{+}\right)+P^{\nu} .
$$

THEOREM. Let $\|\cdot\|$ be some matrix norm. If $\|P\|<1$ and if $P$ is invertible, then $\lim _{\nu \rightarrow \infty} M_{\nu}=M$.

Proof. For all $\nu>1, I+P+P^{2}+\cdots+P^{\nu-1}=(I-P)^{-1}\left(I-P^{\nu}\right)$. If $\|P\|<1$, then $\lim _{\nu \rightarrow \infty}(I-P)^{-1}\left(I-P^{\nu}\right)=(I-P)^{-1}$. Hence,

$$
\begin{aligned}
\lim _{\nu \rightarrow \infty} M_{\nu} & =(I-P)^{-1} Q\left(A_{1}-A_{+}\right)=\left(A_{1} Q\right)^{-1} Q\left(A_{1}-A_{+}\right) \\
& =A_{1}^{-1} Q^{-1} Q\left(A_{1}-A_{+}\right)=M .
\end{aligned}
$$

Local mode analysis, applied to (2.14b) with $A_{1}$ and $A_{+}$according to (2.10) and (2.11), respectively, yields for the maximally allowable value of $\sigma \equiv c \Delta t / \Delta x, \sigma_{v=1}=2.21$ and $\sigma_{\lim \nu \rightarrow \infty}=2.12$. Note that the difference between both values is very small. For an arbitrary $\nu$ it is safe and still efficient to take $\sigma=2.12$. The value $\sigma=2.12$ is lower than that for the existing method applied to the first-order upwind system $(\sigma=2.5105)$, but higher than that for the existing method applied to the higher-order system $(\sigma=1.9186)$.

For $\sigma=2.12$ and for increasing $\nu$, Fig. 4 shows the behavior of the convergence factor $\mu$, versus the frequency $\theta$ in the range $[0, \pi]$, for the new higher-order method. Already for $\nu=1$, it appears that the convergence behavior of the new higher-order method is better than that of the existing higher-order method (Fig. 5). Clearly visible for increasing $\nu$ is the rapid improvement of the smoothing (i.e., the convergence in the range $\theta \in[\pi / 2, \pi])$ and the tendency towards coincidence of the curves. The curves converge to the one that corresponds with the exact solution of $(2.14 \mathrm{~b}): \mu=\frac{1}{2} \sin \theta, \theta \in$ $[0, \pi]$. In the next section we will further investigate the smoothing properties of IDeC.

2.3.2. Smoothing analysis. Local mode analysis yields that with the new method, optimal smoothing of the highest frequency, $\theta=\pi$, is obtained for $\sigma=1.8921$ and $\sigma=1.4869$. Just as for $\sigma=2.12$ (Fig. 4), Figs. 6 and 7 give the convergence behavior for $\sigma=1.8921$ and $\sigma=1.4869$ with increasing $\nu$. For both values of $\sigma$ the smoothing is clearly better than for $\sigma=2.12$. For $\sigma=1.4869$ it is best. 


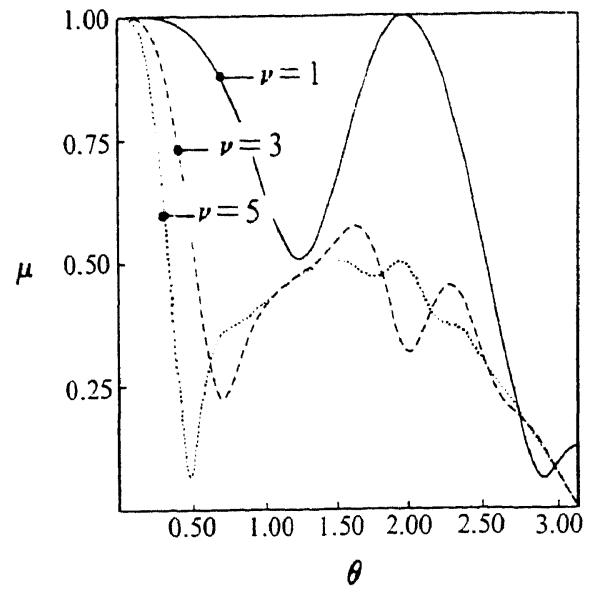

(a)

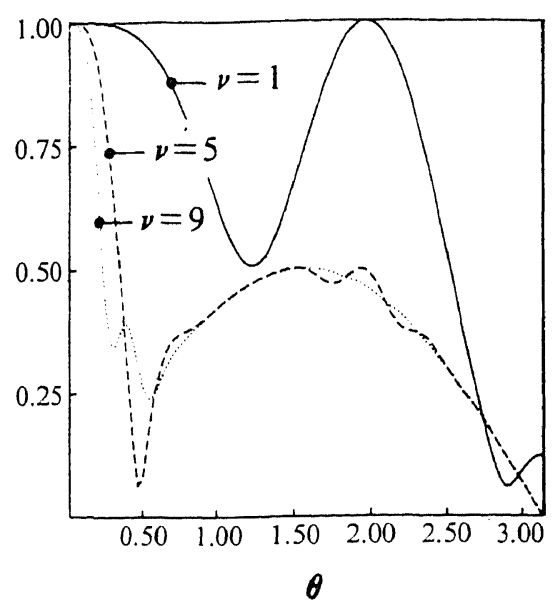

(b)

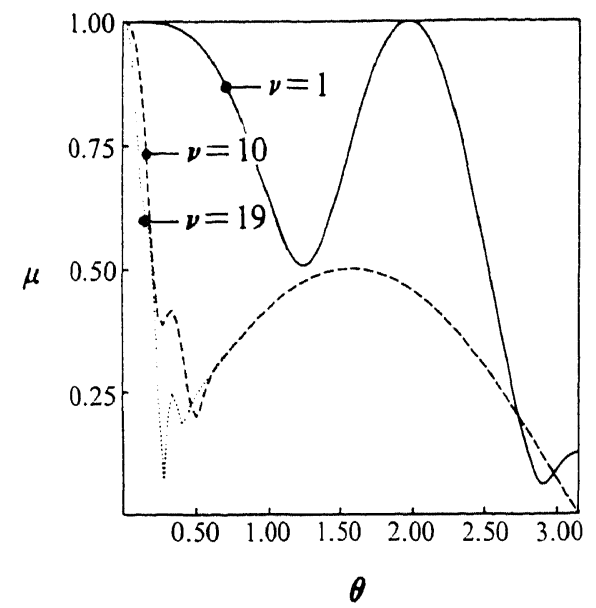

(c)

FIG. 4. Convergence behavior novel higher-order method, for $\sigma=2.12$. (a) $\nu=1,3,5$. (b) $\nu=1,5$, 9. (c) $\nu=1,10,19$.

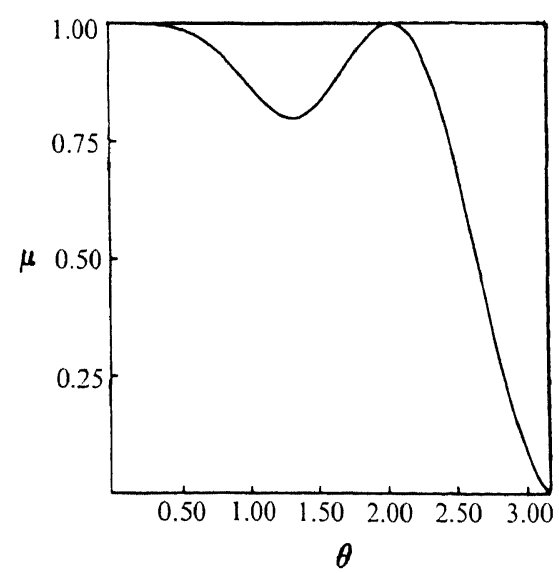

FIG. 5. Convergence behavior existing higher-order method, for $\sigma=1.9186$. 


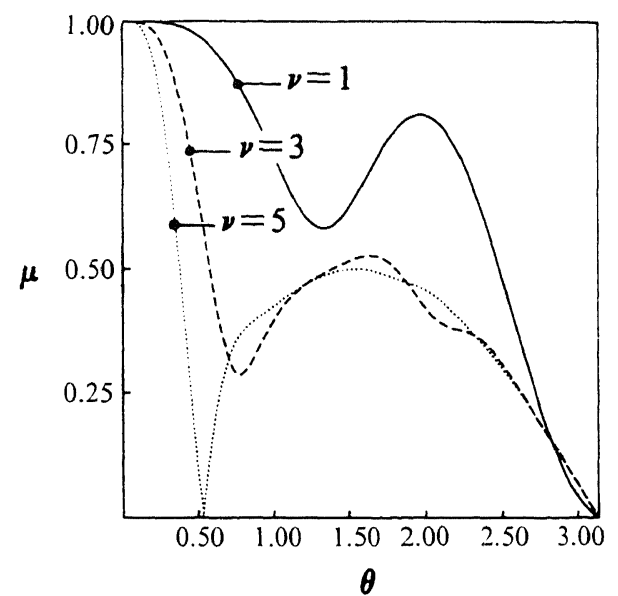

(a)

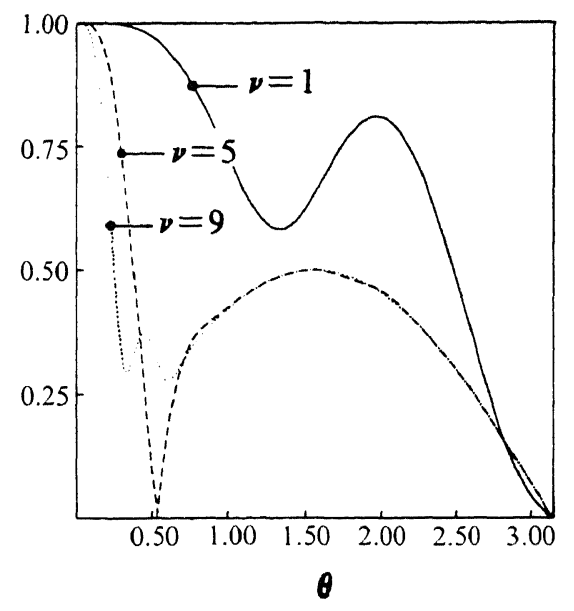

(b)

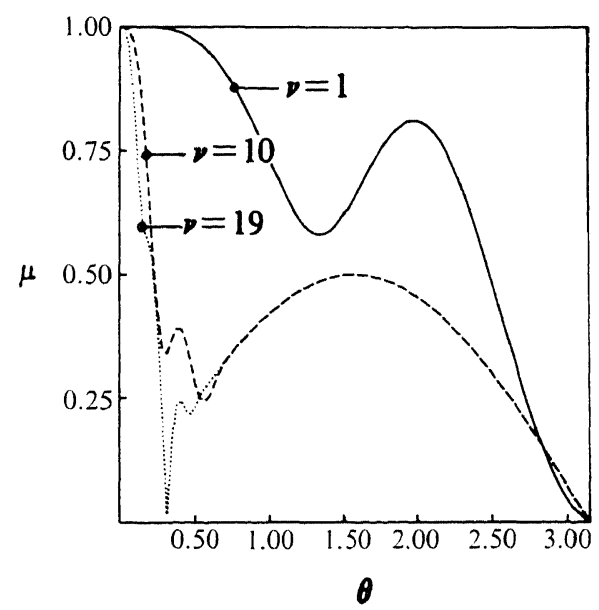

(c)

FIG. 6. Convergence behavior novel higher-order method, for $\sigma=1.8921$. (a) $\nu=1,3,5$. (b) $\nu=1,5,9$. (c) $\nu=1,10,19$.

In Figs. 8, 9, and 10 we show the smoothing behavior for varying $\sigma$, the $\theta$-range considered being $[\pi / 2, \pi]$, and the quantity $\mu$ along the vertical axis being the maximum smoothing factor found over this range. We consider successively: the first-order method (Fig. 8), the existing higher-order method (Fig. 9), and the new higher-order method (Fig. 10). When we compare the results of the existing higher-order method and the new higher-order method (Figs. 9 and 10), we find that the new method clearly has better smoothing properties. The new method appears to have even better smoothing properties than the first-order method (compare Figs. 8 and 10). Note in particular that the $\sigma$-range over which its smoothing is good is much wider. 


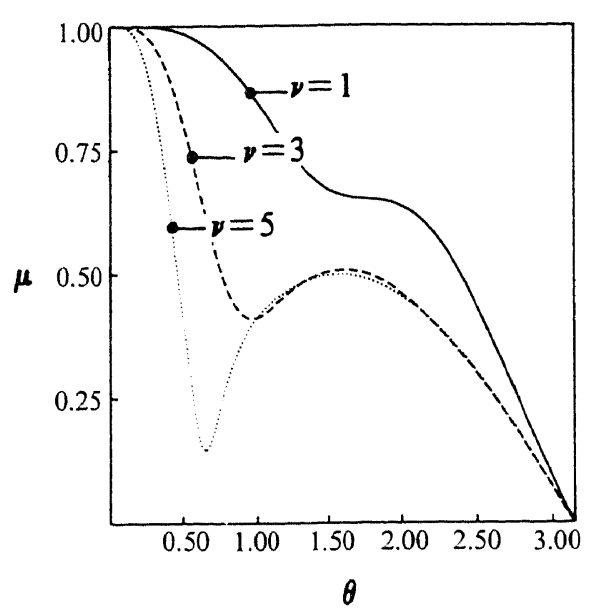

(a)

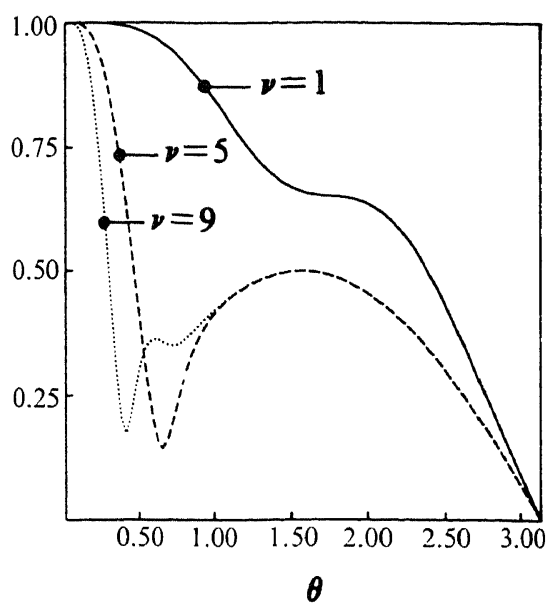

(b)

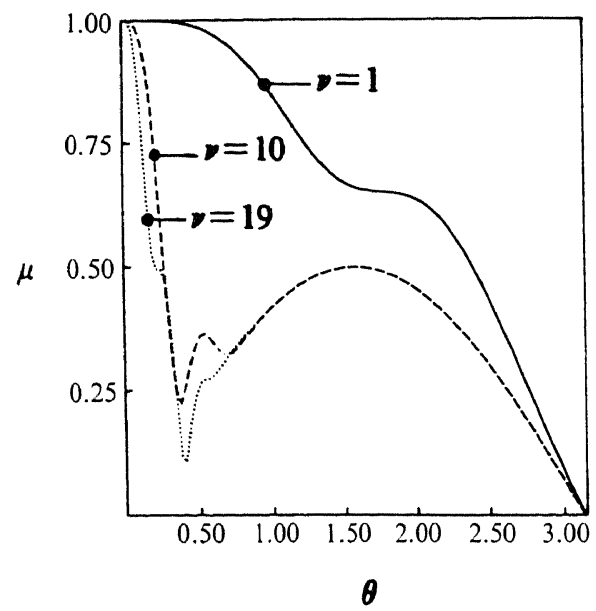

(c)

FIG. 7. Convergence behavior novel higher-order method, for $\sigma=1.4869$. (a) $\nu=1,3,5$. (b) $\nu=1,5,9$. (c) $\nu=1,10,19$.

3. Numerical results. In order to verify the previously predicted better stability and convergence properties of the novel higher-order method, we compute the standard transonic channel flow from [21] with the two-dimensional Euler equations. Three finest grids are considered: a 161-vertices grid (Fig. 11), a 585 -vertices grid that is about twice as fine (see [16]), and a 2225-vertices grid that is about four times as fine. The corresponding solution schedules applied are a four-, five, and six-level schedule $(L=4,5,6)$, respectively, all with $\nu_{\text {pre }}=\nu_{\text {post }}=1$, for all $l$.

In Figs. 12(a)-12(c) we present various convergence histories as obtained for $L=4,5,6$, respectively. The convergence results presented are those of (i) the first-order discretized Euler equations solved by means of the nonlinear multigrid iteration (dotted lines), and those of higher-order discretized Euler equations solved by means of (ii) the existing higher-order method (dashed lines), and (iii) the novel higher-order method 


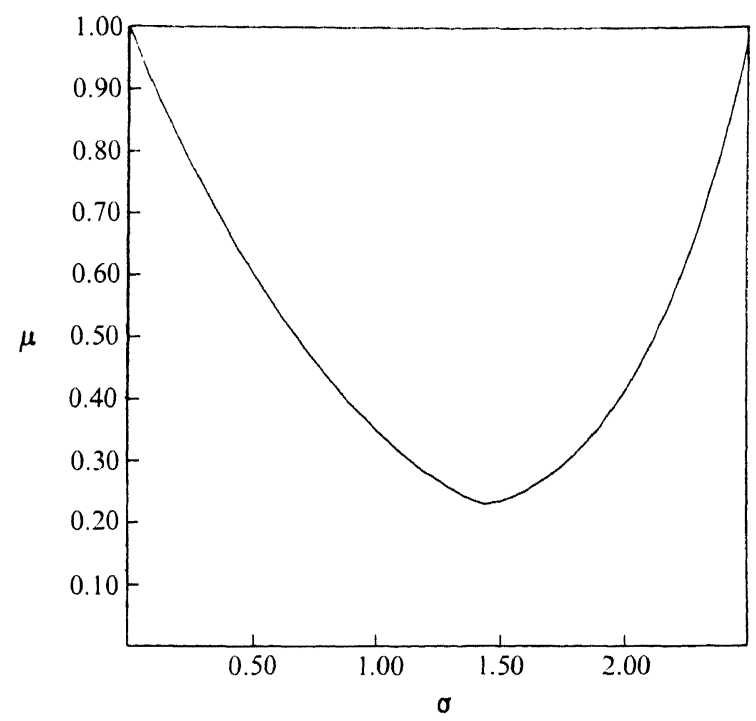

FIG 8. Smoothing behavior for varying $\sigma$, first-order method.

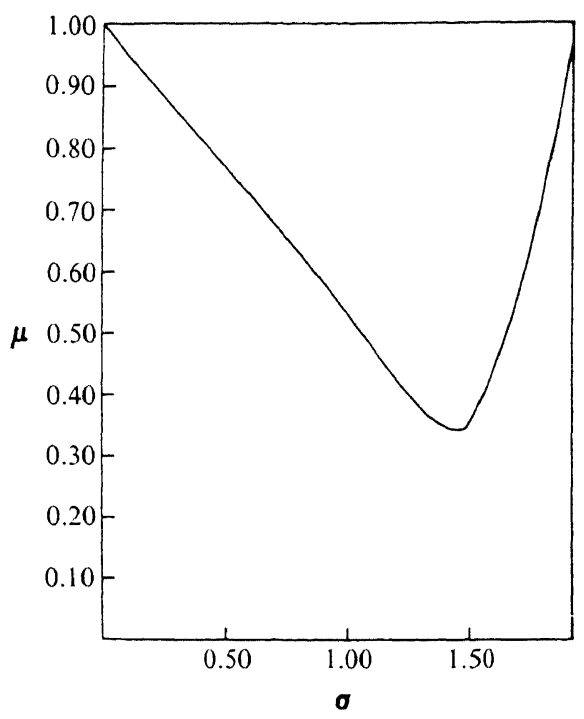

FIG 9. Smoothing behavior for varying $\sigma$, existing higher-order method.

(solid lines). In all three graphs, the residual considered is the $L_{2}$-norm of the error in the conservation of mass over all the finest-grid cells. Furthermore, in all three graphs the number of cycles indicated along the horizontal axis is (i) the number of FAS cycles in case of both the first-order method and the existing higher-order method, and (ii) the number of IDeC cycles in case of the new higher-order method. Note that with the new higher-order method, for $\nu_{\mathrm{FAS}}=2,5,10$ the number of inner FAS cycles is, respectively, 2, 5, and 10 times larger than the number of indicated IDeC cycles. (Only for $\nu_{\mathrm{FAS}}=1$ does the number of FAS cycles equal the number of IDeC cycles.) 


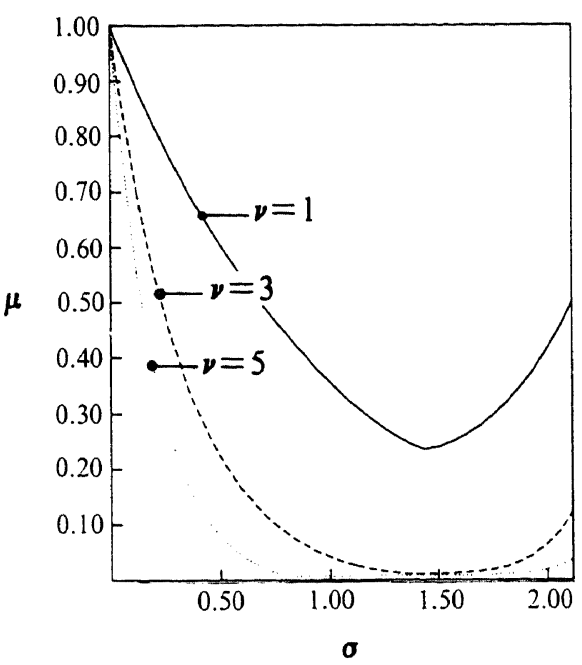

(a)

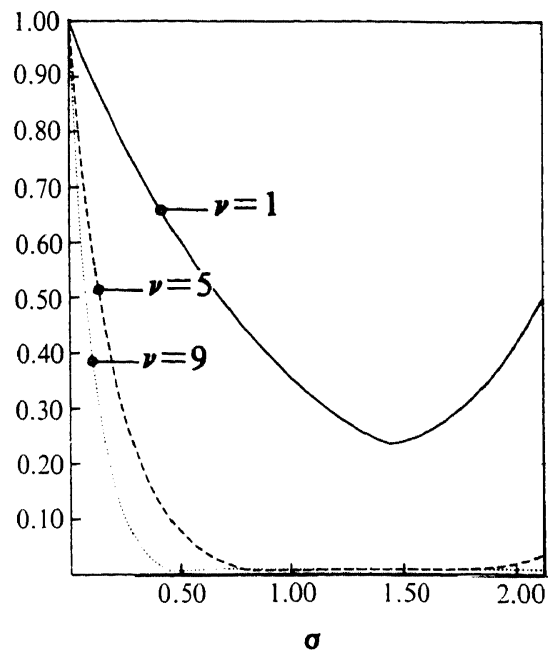

(b)

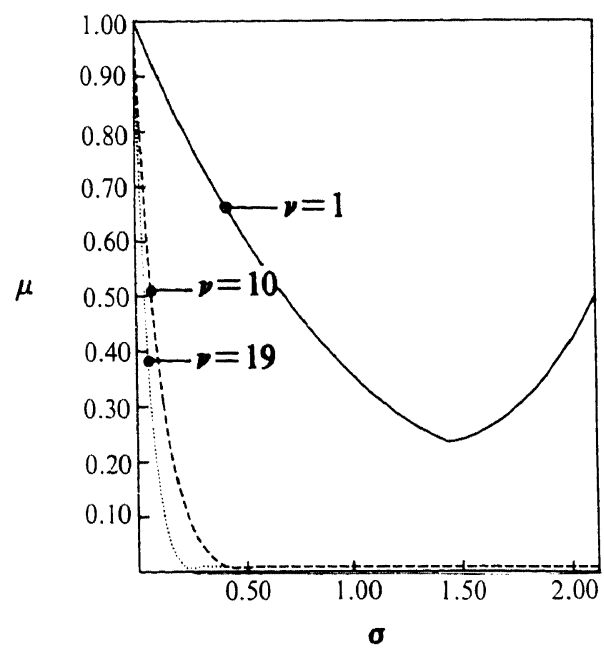

(c)

FIG. 10. Smoothing behavior for varying $\sigma$, novel higher-order method. (a) $\nu=1,3,5$. (b) $\nu=1,5$, 9. (c) $\nu=1,10,19$.

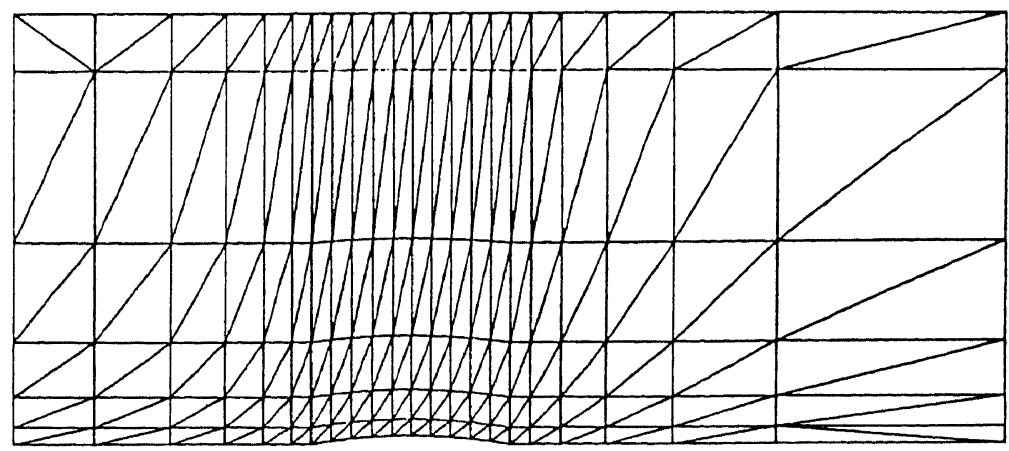

FIG. 11. Channel from [20], with 161 -vertices grid. 


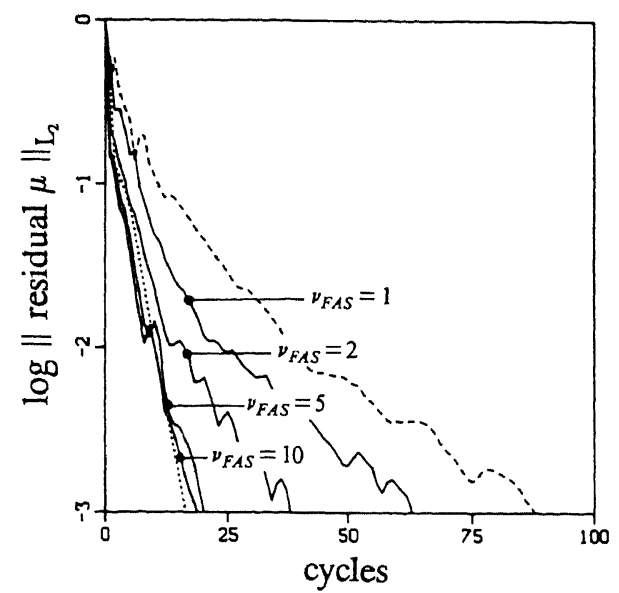

(a)

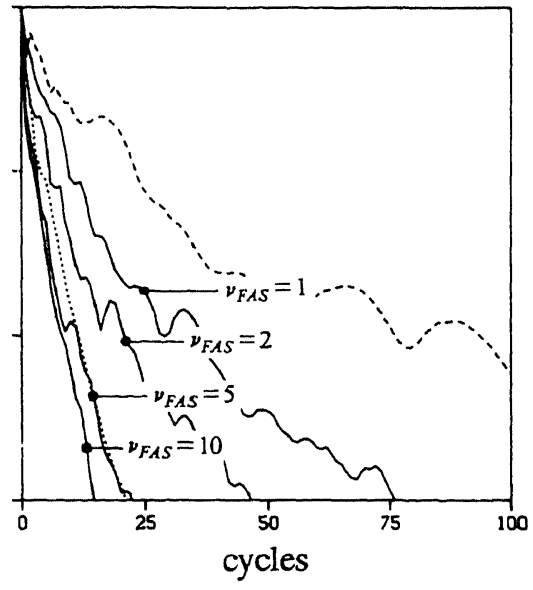

(b)

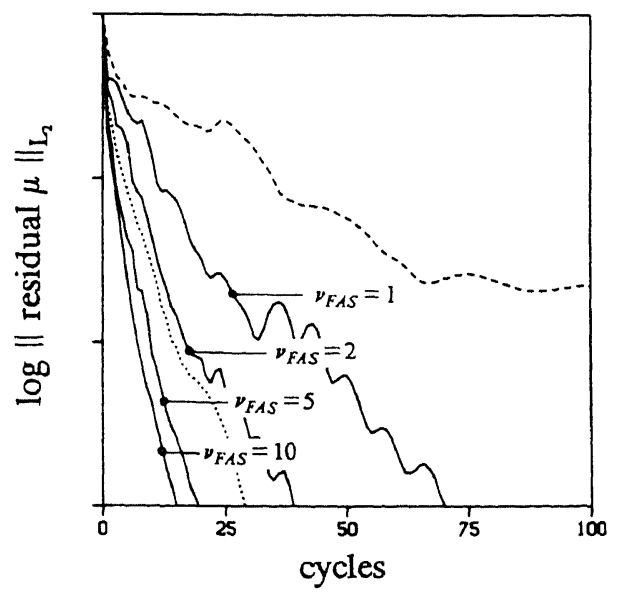

(c)

FIG. 12. Convergence histories: first-order method, ‥; existing higher-order method, - - -; novel higher-order method, _- (a) 161 -vertices grid $(L=4)$. (b) 585-vertices grid $(L=5)$. (c) 2225-vertices grid $(L=6)$.

All convergence histories start at the end of the FMG stage (Fig. 3). In agreement with the theoretical results found in $\S 2.3$, for all four values of $\nu_{\mathrm{FAS}}$ (so also for $\nu_{\mathrm{FAS}}=1$ ), the new method does indeed give a better convergence than the existing higher-order method. For decreasing mesh width, the convergence of the new higher-order method becomes even relatively better than that of the first-order method. (For all four values of $\nu_{\mathrm{FAS}}$ under consideration, the corresponding convergence histories in Fig. 12 show a better grid-independency than those of the multigrid method applied to the first-order discretized equations.) This better performance is probably due to better smoothing in the new method. (In $\S 2.3$, by model analysis, we have found that the new method has better smoothing properties than the first-order method.)

As for the actual order of accuracy, if we took the converged higher-order accurate solution obtained on the 2225 -vertices grid as the reference solution, we measured local orders of accuracy in the range $\left[O\left(h^{1.4}\right), O\left(h^{2.3}\right)\right]$ for the solutions on the coarser 


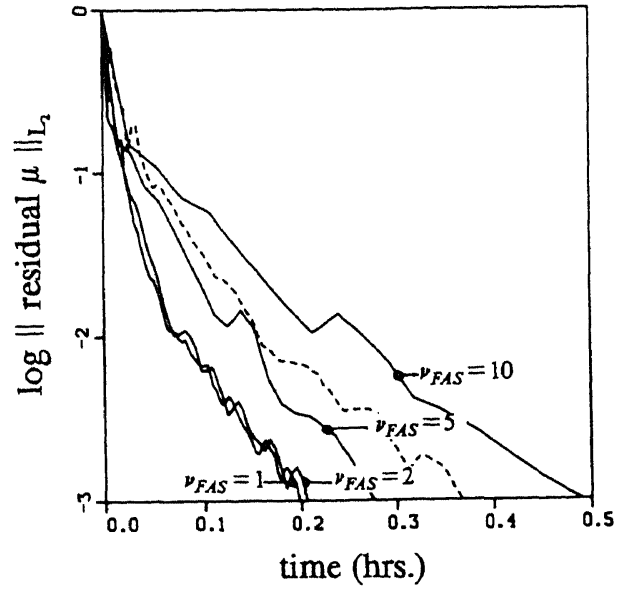

(a)

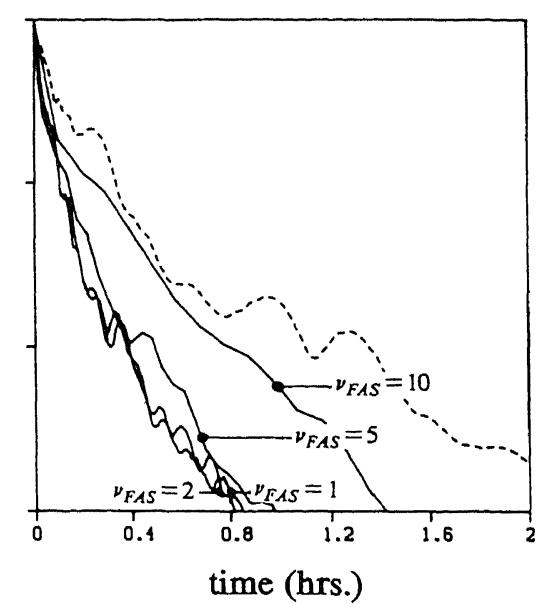

(b)

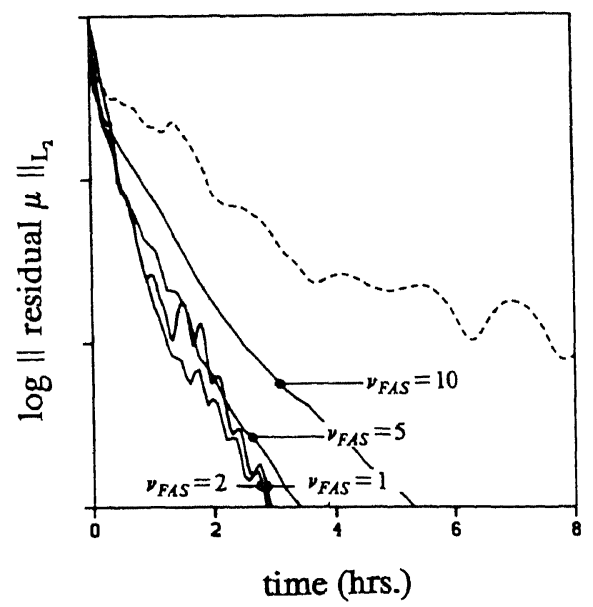

(c)

FIG. 13. Efficiency histories: existing higher-order method,_---; novel higher-order method, (a) 161 -vertices grid $(L=4)$. (b) 585-vertices grid $(L=5)$. (c) 2225-vertices grid $(L=6)$.

grids (the 585-vertices grid and the 161-vertices grid). The global order of accuracy appears to be almost $O\left(h^{2}\right)$.

Finally, the important question of which of the various higher-order methods is the most efficient still remains. To answer this question, we give the higher-order efficiency histories in Figs. 13(a)-13(c). The indicated computing times have been obtained on a Sequent. (No efforts have been undertaken to make efficient use of the parallelization features of the machine. What interests us here is the relative efficiency of the higher-order methods only.) Since the sizes of the three grids considered are related to each other by approximately a factor 4 , we have related the scales along the horizontal axes accordingly. Concerning the relative efficiency of the novel higher-order method, for the four values of $\nu_{\text {FAS }}$ considered, it appears that for all three grids the best efficiency is obtained with $\nu_{\mathrm{FAS}}=1$ (so just as in [14], for the schedule with only a single FAS cycle per IDeC cycle). Furthermore, it is significant that the novel method 
with $\nu_{\mathrm{FAS}}=1$ appears to be more efficient than the existing higher-order method. Due to the better grid-independency of the novel method, this relatively better efficiency becomes even increasingly better with decreasing meshwidth.

4. Conclusions. Fully implicit solution methods for higher-order discretized equations may strongly benefit from iterative defect correction when these systems of discretized equations are not easily invertible, which is often the case with higher-order accurate discretizations. Fully explicit solution methods may also profit from iterative defect correction. Here the profits are faster convergence and higher efficiency. The defect correction method appears to lead to greater stability (and hence to greater robustness) than the existing (standard) explicit method. Compared to the existing explicit method, it possesses remarkably good smoothing properties, in fact even better than the first-order method. Last but not least, its convergence rate appears to be grid-independent. For upwind discretizations, the "price" which has to be paid for using defect correction iteration, a slightly more complex algorithm, is negligible, because of the direct availability of an appropriate approximate operator; the first-order upwind operator.

Acknowledgments. The authors want to thank Joke Sterringa, P. Wesseling, and the referees for their suggestions in improving this paper.

\section{REFERENCES}

[1] K. BABA AND M. TABATA, On a conservative upwind finite element scheme for convective diffusion equations, RAIRO Numer. Anal., 15 (1981), pp. 3-25.

[2] K. BÖHMER, P. W. HEMKER, AND H. J. STETTER, The defect correction approach, Comput. Suppl., 5 (1984), pp. 1-32.

[3] A. BRANDT, Multi-level adaptive solutions to boundary-value problems, Math. Comput., 31 (1977), pp. 330-399.

[4] - Guide to multigrid development, Lecture Notes in Math. 960, Springer-Verlag, Berlin, 1982, pp. 220-312.

[5] A. Dervieux, J.-A. Desideri, F. Fezoui, B. Palmerio, J. P. Rosenblum, and B. Stoufflet, Euler calculations by upwind finite element methods and adaptive mesh algorithms, Notes on Numerical Fluid Mechanics, No. 26, Vieweg, Braunschweig, 1989, pp. 138-156.

[6] J.-A. DESIDERI AND P. W. HEM KER, Analysis of the convergence of iterative implicit and defect-correction algorithms for higher-order problems, Rapport de Recherche 1200, INRIA Sophia-Antipolis, Valbonne, 1990.

[7] L. FEZOUI AND B. STOUFFLET, A class of implicit upwind schemes for Euler simulations with unstructured grids, J. Comput. Phys., 84 (1989), pp. 174-206.

[8] S. K. GoDUNOV, Finite difference method for numerical computation of discontinuous solutions of the equations of fluid dynamics, Mat. Sb., 47 (1959), pp. 271-306. (Cornell Aeronautical Lab. Transl. from Russian.)

[9] W. HACKBUSCH, Bemerkungen zur iterierten Defektkorrektur und zu ihrer Kombination mit Mehrgitterverfahren, Rev. Roumaine Math. Pures Appl., 26 (1981), pp. 1319-1329.

[10] Multi-grid methods and applications, Springer-Verlag, Berlin, 1985.

[11] P. W. HEMKER, Mixed defect correction iteration for the solution of a singular perturbation problem, Comput. Suppl., 5 (1984), pp. 123-145.

[12] Defect correction and higher order schemes for the multi grid solution of the steady Euler equations, Lecture Notes in Math. 1228, Springer-Verlag, Berlin, 1986, pp. 149-165.

[13] A. JAMESON, Solution of the Euler equations for two dimensional transonic flow by a multigrid method, Appl. Math. Comput., 13 (1983), pp. 327-355.

[14] B. KOREN, Defect correction and multigrid for an efficient and accurate computation of airfoil flows, J. Comput. Phys., 77 (1988), pp. 183-206.

[15] - Multigrid and defect correction for the steady Navier-Stokes equations, J. Comput. Phys., 87 (1990), pp. 25-46. 
[16] M.-H. Lallemand AND A. DeRvieux, A multigrid finite element method for solving the two-dimensional Euler equations, Lecture Notes in Pure and Appl. Math. 110, Marcel Dekker, New York, 1988, pp. 337-363.

[17] M.-H. LALlEMAND, Dissipative properties of Runge-Kutta schemes with upwind spatial approximation for the Euler equations, Rapport de Recherche 1179, INRIA Sophia-Antipolis, Valbonne, 1990.

[18] B. VAN LEER, Towards the ultimate conservative difference scheme V. A second-order sequel to Godunov's method, J. Comput. Phys., 32 (1979), pp. 101-136.

[19] - Upwind-difference methods for aerodynamic problems governed by the Euler equations, Lectures in Appl. Math. 22, Part 2, Amer. Math. Soc., Providence, RI, 1985, pp. 327-336.

[20] S. OSHER AND F. SOLOMON, Upwind-difference schemes for hyperbolic systems of conservation laws, Math. Comput., 38 (1982), pp. 339-374.

[21] A. RIZZI AND H. VIVIAND, Numerical methods for the computation of inviscid transonic flows with shock waves, Notes on Numerical Fluid Mechanics, No. 3, Vieweg, Braunschweig, 1981.

[22] P. L. ROE, Approximate Riemann solvers, parameter vectors and difference schemes, J. Comput. Phys., 43 (1981), pp. 357-372.

[23] P. ROSTAND AND B. STOUFFLET, TVD schemes to compute compressible viscous flows on unstructured meshes, Notes on Numerical Fluid Mechanics, No. 24, Vieweg, Braunschweig, 1989, pp. 510-520. 